\title{
OUTER MEASURES, MEASURABILITY, AND LATTICE REGULAR MEASURES
}

\author{
JAMES PONNLEY \\ Department of Mathematics \\ Medgar Evers College \\ The City University of New York \\ 1650 Bedford Avenue \\ Brooklyn, New York 11245-2298 \\ and \\ Department of Mathematical Sciences \\ Clark Atlanta University \\ Atlanta, GA 30314
}

(Received September 28, 1994 and in revised form February 6, 1995)

\begin{abstract}
Let $X$ be an arbitrary non-empty set, and $\mathcal{L}$ a lattice of subsets of $X$ such that $\emptyset, X \in \mathcal{L} \mathcal{A}(\mathcal{L})$ denotes the algebra generated by $\mathcal{L}$ and $I(\mathcal{L})$ those zero-one valued, non-trivial, finitely additive measures on $\mathcal{A}(\mathcal{L}) \quad I_{\sigma}(\mathcal{L})$ denotes those elements of $I(\mathcal{L})$ that are $\sigma$-smooth on $\mathcal{L}$, and $I_{R}(\mathcal{L})$ denotes those elements of $I(\mathcal{L})$ that are $\mathcal{L}$-regular while $I_{R}^{\sigma}(\mathcal{L})=I_{R}(\mathcal{L}) \cap I_{\sigma}(\mathcal{L}) \quad$ In terms of those and other subsets of $I(\mathcal{L})$, various outer measures are introduced, and their properties are investigated Also, the interplay between the measurable sets associated with these outer measures, regularity properties of the measures, smoothness properties of the measures, and lattice topological properties are thoroughly investigated - yielding new results for regularity or weak regularity of these measures, as well as domination on a lattice of a suitably given measure by a regular one Finally, elements of $I_{o}(\mathcal{L})$ are fully characterized in terms of induced measures on a certain generalized Wallman space
\end{abstract}

KEY WORDS AND PHRASES. Associated outer measures, measurable sets, weakly regular measures, slightly regular measures, almost normal lattices, domination by regular measures

1991 AMS SUBJECT CLASSIFICATION CODES. 28C15, 28A12

\section{INTRODUCTION.}

Let $X$ be an arbitrary non-empty set, and $\mathcal{L}$ a lattice of subsets of $X$ such that $X, \emptyset \in \mathcal{L} . \mathcal{A}(\mathcal{L})$ denotes the algebra generated by $\mathcal{L}$, and $I(\mathcal{L})$ denotes those zero-one valued, non-trivial finitely additive measures on $\mathcal{A}(\mathcal{L}) \quad I_{\sigma}(\mathcal{L})$ denotes the set of $\mu \in I(\mathcal{L})$ that are $\sigma$-smooth on $\mathcal{L}$, that is, if $L_{n} \in \mathcal{L}$, for all $n$, and if $L_{n} \downarrow \emptyset$, then $\mu\left(L_{n}\right) \rightarrow 0$. J(L) denotes the strongly $\sigma$-smooth elements of $I(\mathcal{L})$, that is, those $\mu \in I(\mathcal{L})$ such that if $L_{n} \downarrow L$, where $L_{n}, L \in \mathcal{L}$, then $\mu\left(L_{n}\right) \rightarrow \mu(L) . I^{\sigma}(\mathcal{L})$ denotes those $\mu \in I(\mathcal{L})$ which are $\sigma$-smooth on $\mathcal{A}(\mathcal{L})$, which is equivalent here to $\mu$ being countably additive $I_{R}(\mathcal{L})$ denotes those $\mu \in I(\mathcal{L})$ which are $\mathcal{L}$-regular, and $I_{R}(\mathcal{L}) \cap I_{\sigma}(\mathcal{L})=I_{R}^{\sigma}(\mathcal{L}) \quad$ Further specialized subsets of measures are introduced in Sections 3 and 4

Associated with these measures are certain outer measures (finitely or countably subadditive) $\mu^{\prime}, \mu^{\prime \prime}$, $\tilde{\mu}, \tilde{\tilde{\mu}}$ We investigate the behavior of these outer measures on both $\mathcal{L}$ and $\mathcal{L}^{\prime}$, the complementary lattice to $\mathcal{L}$ and other related lattices to characterize the various specialized sets of measures, and thereby extend the results given in $[5,6,7]$ We also consider the interplay of the lattice $\mathcal{L}$ with the measurable sets of some of these outer measures

In Section 4 we use these results to obtain conditions for a $\mu \in I_{\sigma}(\mathcal{L})$ or $J(\mathcal{L})$ to be dominated on $\mathcal{L}$ by a $\nu \in I_{R}^{\sigma}(\mathcal{L})$ or to be equal to a $\nu \in I_{R}^{\sigma}(\mathcal{L})$ Since some of these results can be expressed in terms of generalized Wallman spaces, we close Section 4 with a brief look at one of these spaces 
We give a review in Section 2 of the notation to be used, and of some standard lattice-measure theoretic results. Related matters can be found in $[2,4,5,6]$

\section{BACKGROUND AND NOTATIONS}

In this section, we introduce the relevant notation and terminology that will be used throughout the paper. All of this is fairly standard and is consistent with [1,3,6,8]; we include it for the reader's convenience. We also include in this section several recent results as well as several new results pertaining to various induced outer measures.

$X$ will denote throughout, an arbitrary non-empty set, and $\mathcal{L}$ a lattice of subsets such that $\emptyset, X \in \mathcal{L}$. $\mathcal{A}(\mathcal{L})$ will denote the algebra generated by $\mathcal{L}$, and $I(\mathcal{L})$ those non-trivial zero-one valued, finitely additive measures on $\mathcal{A}(\mathcal{L})$.

$I_{\sigma}(\mathcal{L})$ denotes those elements of $I(\mathcal{L})$ that are $\sigma$-smooth on $\mathcal{L}$, namely, if $\mu \in I_{\sigma}(\mathcal{L})$ then $L_{n} \downarrow \emptyset$, $L_{n} \in \mathcal{L}$, implies $\mu\left(L_{n}\right) \rightarrow 0 . J(\mathcal{L})$ denotes those elements of $I(\mathcal{L})$ that are strongly $\sigma$-smooth on $\mathcal{L}$, i.e., if $L_{n} \downarrow L$, where $L_{n} \in \mathcal{L}$ and $L \in \mathcal{L}$, then $\mu\left(L_{n}\right) \rightarrow \mu(L) . I^{\sigma}(\alpha)$ denotes those $\mu \in I(\mathcal{L})$ which are $\sigma$ smooth on $\mathcal{A}(\mathcal{L})$ or, equivalently, are countably additive. $I_{R}(\mathcal{L})$ denotes those $\mu \in I(\mathcal{L})$ which are $\mathcal{L}$ regular, namely

$$
\mu(A)=\sup \{\mu(L) \mid L \subset A, L \in \mathcal{L}\},
$$

where $A \in \mathcal{A}(\mathcal{L})$. It is easy to see that if $\mu \in I_{\sigma}(\mathcal{L})$ and if $\mu \in I_{R}(\mathcal{L})$, then $\mu \in I^{\sigma}(\mathcal{L})$; we denote these elements by $I_{R}^{\sigma}(\mathcal{L})$.

For $\mu \in I(\mathcal{L}), \mathcal{S}(\mu)$ denotes the support of $\mu$, and is given by

$$
\mathcal{S}(\mu)=\cap\{L \in \mathcal{L} \mid \mu(L)=1\} .
$$

Next, we denote by $\mathcal{L}^{\prime}$ the complementary lattice of $\mathcal{L}$, i.e., $\mathcal{L}^{\prime}=\left\{L^{\prime} \mid L \in \mathcal{L}\right\}$, and where the prime stands for complement.

$\delta(\mathcal{L})$ : lattice of all countable intersections of sets of $\mathcal{L}$. $\mathcal{L}$ is a delta lattice if $\delta(\mathcal{L})=\mathcal{L}$, i.e., if $\mathcal{L}$ is closed under countable intersections. Now, for $\mu \in I(\mathcal{L})$, define for any set $E \subset X$

$$
\mu^{\prime}(E)=\inf \left\{\mu\left(L^{\prime}\right): E \subset L^{\prime}, L \in \mathcal{L}\right\} .
$$

Clearly, $\mu^{\prime}$ is zero-one valued, $\mu^{\prime}(X)=1, \mu^{\prime}(\emptyset)=0, \mu^{\prime}$ is monotone, and $\mu^{\prime}$ is finitely subadditive. We will sometimes refer to $\mu^{\prime}$ as a finitely subadditive outer measure. Similarly, we define $\tilde{\mu}(E)$ by taking the covering class to be $\mathcal{L}$ instead of $\mathcal{L}^{\prime}$.

Also, let

$$
\mu^{\prime \prime}(E)=\inf \left\{\sum_{i=1}^{\infty} \mu\left(L_{\imath}^{\prime}\right): E \subset \bigcup_{1}^{\infty} L_{i}^{\prime}, L_{i} \in \mathcal{L}\right\} .
$$

Then $\mu^{\prime \prime}$ is a zero-one valued outer measure with $\mu^{\prime \prime}(X)=1$ if $\mu \in I_{\sigma}(\mathcal{L})$. We note, if $\mu \notin I_{\sigma}(\mathcal{L})$, then there exists a sequence $L_{n} \in \mathcal{L}, L_{n} \downarrow \emptyset$, and $\mu\left(L_{n}\right)=1$ all $n$; hence, $\cup L_{n}^{\prime}=X$ and $\mu\left(L_{n}^{\prime}\right)=0$ all $n$, so, $\mu^{\prime \prime}(X)=0$, and consequently, $\mu^{\prime \prime} \equiv 0$. For this reason, when dealing with $\mu^{\prime \prime}$, we usually assume that $\mu \in I_{\sigma}(\mathcal{L})$. Likewise, we can consider $\tilde{\tilde{\mu}}$ where the countable covering class is now taken to be $\mathcal{L}$. Clearly, analogous statements hold for $\tilde{\mu}$.

We will write for either measures or outer measures $\mu \leq \nu(\mathcal{L})$ if $\mu(L) \leq \nu(L)$ for all $L \in \mathcal{L}$. It is now easy to see that if $\mu \in I_{\sigma}(\mathcal{L})$, then

$$
\begin{gathered}
\mu \leq \mu^{\prime \prime} \leq \mu^{\prime}(\mathcal{L}) \quad \text { and } \quad \mu^{\prime \prime} \leq \mu=\mu^{\prime}\left(\mathcal{L}^{\prime}\right) . \quad \text { Also, } \\
\tilde{\mu} \leq \mu=\tilde{\mu} \leq \mu^{\prime \prime} \leq \mu^{\prime}(\mathcal{L})
\end{gathered}
$$

And if $\mu \in I_{\sigma}\left(\mathcal{L}^{\prime}\right)$, then

$$
\mu^{\prime \prime} \leq \mu=\mu^{\prime} \leq \tilde{\mu} \leq \tilde{\mu}\left(\mathcal{L}^{\prime}\right)
$$

We recall that if $\nu$ is an outer measure (finitely subadditive or countably subadditive), then 
$\mathcal{S}_{\nu}$ the $\nu$-measurable sets $=\left\{E \subset X \mid \nu(G)=\nu(G \cap E)+\nu\left(G \cap E^{\prime}\right)\right\}$ for all $G \subset X$ We then have for $\mu \in I(\mathcal{L})$

$$
\mathcal{S}_{\mu^{\prime}}=\left\{E \subset X \mid E \supset L \in \mathcal{L}, \mu(L)=1 \quad \text { or } \quad E^{\prime} \supset L \in \mathcal{L}, \mu(L)=1\right\} .
$$

For $\mathcal{S}_{\widetilde{\mu}}$, we need just replace $L$ by $L^{\prime}$ and $\mathcal{L}$ by $\mathcal{L}^{\prime}$.

Next, for $\mu \in I_{\sigma}(\mathcal{L})$,

$$
\begin{gathered}
S_{\mu^{\prime \prime}}=\left\{E \subset X \mid E \supset \bigcap_{n=1}^{\infty} L_{n}, L_{n} \in \mathcal{L}, \mu\left(L_{n}\right)=1, \quad \text { all } n\right. \\
\left.\quad \text { or } \quad E^{\prime} \supset \bigcap_{n=1}^{\infty} L_{n}, L_{n} \in \mathcal{L}, \mu\left(L_{n}\right)=1, \text { all } n\right\} .
\end{gathered}
$$

The corresponding statement for $\mathcal{S}_{\widetilde{\mu}}$ is clear

Various lattice topological properties such as compact, countably compact, normal, regular, etc. have been characterized in a measure theoretic way, see [6]. We note a few of these here, but, instead, give characterizations in terms of the above outer measures.

(a) $\mathcal{L}$ is $T_{2}$ iff for any $\mu \in I(\mathcal{L})$ one of the following is true

(i) $\mu^{\prime}(\{x\})=0$ for all $x \in X$

(ii) $\mu^{\prime}(\{x\})=1$ for some $x \in X$, and $\mu^{\prime}(\{y\})=0$ for all $y \neq x, y \in X$

(b) $\mathcal{L}$ is compact iff for any $\mu \in I(\mathcal{L})$, there exists an $x \in X$ such that $\mu^{\prime}(\{x\})=1$.

(c) $\mathcal{L}$ is regular iff for any $\mu \in I(\mathcal{L}), \mathcal{S}(\mu)=\mathcal{S}\left(\mu^{\prime}\right)$, where $\mathcal{S}\left(\mu^{\prime}\right)$ is defined in the obvious way with respect to $\mathcal{L}$.

(d) $\mathcal{L}$ is normal iff for $\mu, \nu \in I(\mathcal{L}), \mu \leq \nu(\mathcal{L})$ then $\mu^{\prime}=\nu^{\prime}(\mathcal{L})$.

(e) $\mathcal{L}$ is countably compact iff for every $\mu \in I(\mathcal{L}), \mu^{\prime \prime} \not \equiv 0$.

The following theorem $(b, c)$ is generally well-known, see [4], and we just state it without proof; while (a) is clearly true

\section{THEOREM 2.1.}

(a) If $\mu \in I(\mathcal{L})$, and if $\mathcal{L}$ is countably compact then $\mu^{\prime \prime}=\mu^{\prime}(\mathcal{L})$.

(b) If $\mu \in I_{\sigma}(\mathcal{L})$, and if $\mathcal{L}$ is $\delta$-lattice which is normal then $\mu^{\prime \prime}=\mu^{\prime}(\mathcal{L})$.

(c) If $\mu \in I_{\sigma}(\mathcal{L})$, and if $\mathcal{L}$ is normal and countably paracompact (c.p.), then $\mu^{\prime}=\mu^{\prime \prime}(\mathcal{L})$.

The next theorem is less well-known in the form given, so we provide a proof.

THEOREM 2.2. If $\mathcal{L}$ is complement generated (c.g.) and if $\mu \in I_{\sigma}\left(\mathcal{L}^{\prime}\right)$ then $\widetilde{\mu}=\mu=\mu^{\prime}(\mathcal{L})$ which implies $\mu \in I_{R}^{\sigma}(\mathcal{L})$.

PROOF. $\mu \in I_{\sigma}\left(\mathcal{L}^{\prime}\right)$ implies that $\mu \in I_{\sigma}(\mathcal{L})$ since $\mathcal{L}$ is complement generated, and, therefore c.p. Thus $\widetilde{\mu} \leq \mu=\widetilde{\mu} \leq \mu^{\prime \prime} \leq \mu^{\prime}(\mathcal{L})$ by (2.2). Suppose there exists an $L \in \mathcal{L}$ such that $\widetilde{\mu}(L)=0$, and $\mu^{\prime}(L)=1$. Since $\mathcal{L}$ is c.g., $L=\bigcap_{n=1}^{\infty} L_{n}^{\prime}, L_{n} \in \mathcal{L}$ for all $n$. Then $\mu\left(L_{n}^{\prime}\right)=1$ all $n$. From which we get that $\widetilde{\mu}\left(L^{\prime}\right)=0$, but $\widetilde{\mu}(L)=0$. Hence, $\widetilde{\mu}(X)=0$, a contradiction, since $\mu \in I_{\sigma}\left(\mathcal{L}^{\prime}\right)$. Thus, $\widetilde{\tilde{\mu}}=\mu=\mu^{\prime}(\mathcal{L})$ which clearly implies that $\mu \in I_{R}^{\sigma}(\mathcal{L})$.

The following theorem is known (see [4]).

THEOREM 2.3. If $\mu \in I_{\sigma}(\mathcal{L})$ then $\mu^{\prime \prime}=\mu^{\prime}=\mu\left(\mathcal{L}^{\prime}\right)$ iff $\mu \in J(\mathcal{L})$.

Replacing $\mathcal{L}$ by $\mathcal{L}^{\prime}$, we have that if $\mu \in I_{\sigma}\left(\mathcal{L}^{\prime}\right)$ then $\widetilde{\mu}=\mu=\widetilde{\mu}(\mathcal{L})$ iff $\mu \in J\left(\mathcal{L}^{\prime}\right)$. Clearly, such dual statements can be obtained in general, and we will not bother to point this out in the future except for certain important cases.

We close this section with a simple but useful observation, namely,

$$
I_{\sigma}\left(\mathcal{L}^{\prime}\right) \subset I_{\sigma}(\mathcal{L}) \quad \text { iff for all } \quad \mu \in I_{\sigma}\left(\mathcal{L}^{\prime}\right), \widetilde{\mu} \leq \mu^{\prime \prime}(\mathcal{L}) .
$$

Indeed, if $I_{\sigma}\left(\mathcal{L}^{\prime}\right) \subset I_{\sigma}(\mathcal{L})$, then $\widetilde{\mu} \leq \mu \leq \mu^{\prime \prime}(\mathcal{L})$ by $(2.1)$. Conversely, if for $\mu \in I_{\sigma}\left(\mathcal{L}^{\prime}\right)$, $\widetilde{\mu} \leq \mu^{\prime \prime}(\mathcal{L})$, then $1=\widetilde{\widetilde{\mu}}(X)=\mu^{\prime \prime}(X)$, and $\mu \in I_{\sigma}(\mathcal{L})$. 


\section{APPLICATIONS OF THE ASSOCIATED OUTER MEASURES}

We continue in this section to study the applications of $\mu^{\prime}, \mu^{\prime \prime}, \tilde{\mu}$ and $\widetilde{\mu}$ We first recall from [5].

DEFINITION 3.1. $\mu \in I_{S}(\mathcal{L})$ if $\mu \in I_{\sigma}(\mathcal{L})$, and $\mu\left(L^{\prime}\right)=1, L \in \mathcal{L}$, implies there exists $L_{n} \in \mathcal{L}$, $n=1,2, \ldots$ such that $\mu\left(L_{n}\right)=1$, all $n$, and $L^{\prime} \supset \bigcap_{n=1}^{\infty} L_{n}$. Such a measure $\mu$ is often referred to as being slightly regular It is known that (see [5]).

THEOREM 3.1. (a) If $\mu \in I_{S}(\mathcal{L})$ then $\mathcal{L} \subset \mathcal{S}_{\mu^{\prime \prime}}, \mu=\mu^{\prime \prime}(\mathcal{L})$ and $\mu \in I^{\sigma}(\mathcal{L})$.

(b) If $\mu=\mu^{\prime \prime}(\mathcal{L})$ then $\mathcal{L} \subset \mathcal{S}_{\mu^{\prime \prime}}$, and $\mu \in I_{S}(\mathcal{L})$.

We continue in this spirit. First we show

THEOREM 3.2. Let $\mu \in I_{\sigma}(\mathcal{L}) \cap I_{\sigma}(\mathcal{L})$. Then $\widetilde{\tilde{\mu}}=\mu^{\prime \prime}(\mathcal{L})$ implies that

$$
\mathcal{S}_{\widetilde{\mu}} \cap \mathcal{L} \subset \mathcal{S}_{\mu^{\prime \prime}} \cap \mathcal{L} .
$$

PROOF. Let $L \in \mathcal{S}_{\widetilde{\mu}} \cap \mathcal{L}$. Then by the hypothesis and by (2.3),

$$
\widetilde{\mu}(X)=\widetilde{\mu}(L)+\widetilde{\mu}\left(L^{\prime}\right) \geq \mu^{\prime \prime}(L)+\mu^{\prime \prime}\left(L^{\prime}\right) \geq \mu^{\prime \prime}(X) .
$$

But $\widetilde{\mu}(X)=\mu^{\prime \prime}(X)=\mu(X)$ since $\mu \in I_{\sigma}(\mathcal{L}) \cap I_{\sigma}\left(\mathcal{L}^{\prime}\right)$, and by the regularity of $\mu^{\prime \prime}, L \in \mathcal{S}_{\mu^{\prime \prime}} \cap \mathcal{L}$.

REMARKS 3.1. We recall that an outer measure $\nu$ (finitely or countably subadditive) is regular if for any $E \subset X$ there exists an $M \in \mathcal{S}_{\nu}$ such that $E \subset M$, and $\nu(E)=\nu(M)$. Clearly, if $\nu$ is just zeroone valued, then $\nu$ is regular.

THEOREM 3.3. $\mu \in I_{R}^{\sigma}(\mathcal{L})$ implies that $\mu^{\prime \prime} \leq \widetilde{\mu}$, and, consequently $\mathcal{S}_{\widetilde{\mu}} \subset \mathcal{S}_{\mu^{\prime \prime}}$.

PROOF. Let $E \subset X$, and $\mu^{\prime \prime}(E)=1$. If $\widetilde{\mu}(E)=0$, then there exists $A_{\imath} \in \mathcal{L}$ such that $E \subset \bigcup_{1}^{\infty} A_{\imath}, \mu\left(A_{\imath}\right)=0$ all $i$. Since $\mu \in I_{R}(\mathcal{L}), \mu\left(A_{\imath}\right)=0$ implies

$$
A_{\imath} \subset L_{\imath}^{\prime} \in \mathcal{L}^{\prime}, \mu\left(L_{\imath}^{\prime}\right)=0 .
$$

Therefore, $E \subset \bigcup_{1}^{\infty} L_{2}^{\prime}, \mu\left(L_{\imath}^{\prime}\right)=0$, so, $\mu^{\prime \prime}(E)=0$, a contradiction. Thus, $\mu^{\prime \prime} \leq \widetilde{\mu}$.

The following theorem is easy to prove, and we will just state it.

THEOREM 3.4. $\mu \in J(\mathcal{L})$ if and only if for $L_{n} \in \mathcal{L}, L_{n} \downarrow, \tilde{\mu}\left(\cap L_{n}\right)=\inf \mu\left(L_{n}\right)$. If, however, $\mu \in I_{S}(\mathcal{L})$, we have:

THEOREM 3.5. $\mu \in I_{S}(\mathcal{L})$ implies $\widetilde{\mu}\left(\cap L_{n}\right)=$ inf $\widetilde{\mu}\left(L_{n}\right)$, where $L_{n} \in \mathcal{L}, L_{n} \downarrow$, and $\widetilde{\mu}=\tilde{\mu}(\delta(\mathcal{L}))$.

PROOF. Since $\mu \in I_{S}(\mathcal{L}), \mu \in I^{\sigma}(\mathcal{L})$ by Theorem 3.1(a). Hence, $\mu \in J(\mathcal{L}) \cap J\left(\mathcal{L}^{\prime}\right)$. Thus, $\mu=\widetilde{\mu}=\widetilde{\mu}(\mathcal{L})$ since $\mu \in J\left(\mathcal{L}^{\prime}\right)$, and since

$$
\mu \in J(\mathcal{L}), \tilde{\mu}\left(\cap L_{n}\right)=\inf \mu\left(L_{n}\right) \geq \inf \tilde{\mu}\left(L_{n}\right),
$$

using Theorem 3.4. Now, in general, $\widetilde{\mu} \leq \tilde{\mu}$. We show $\widetilde{\mu}=\widetilde{\mu}(\delta(\mathcal{L}))$. Suppose $\widetilde{\mu}\left(\bigcap_{1}^{\infty} L_{n}\right)=0$, but $\tilde{\mu}\left(\bigcap_{1}^{\infty} L_{n}\right)=1, L_{n} \in \mathcal{L}$. Then $\bigcap_{1}^{\infty} L_{n} \subset \bigcup_{1}^{\infty} A_{m}, A_{m} \in \mathcal{L}$ and $\mu\left(A_{m}\right)=0$ all $m$. But $\mu\left(L_{n}\right)=1$ since $\tilde{\mu}\left(\cap L_{n}\right)=1$. Thus,

$\bigcup_{1}^{\infty} L_{n}^{\prime} \supset \bigcap_{1}^{\infty} A_{m}^{\prime}, \mu\left(L_{n}^{\prime}\right)=0 \quad$ all $n$.

Consequently, $\mu^{\prime \prime}\left(\bigcap_{1}^{\infty} A_{m}^{\prime}\right)=0$ which implies here that $1=\mu^{\prime \prime}\left(\cup A_{m}\right) \leq \sum \mu^{\prime \prime}\left(A_{m}\right)$.

Hence, $\mu^{\prime \prime}\left(A_{m}\right)=1$ for some $m$. But $\mu^{\prime \prime}\left(A_{m}\right)=\mu\left(A_{m}\right)$ since $\mu \in I_{S}(\mathcal{L})$. However, $\mu^{\prime \prime}\left(A_{m}^{\prime}\right)=\mu\left(A_{m}^{\prime}\right)=1$, all $m$, since $\mu \in J(\mathcal{L})$ (see Theorem 2.3). Thus, we have a contradiction, and, therefore, $\widetilde{\mu}=\widetilde{\mu}(\delta(\mathcal{L}))$, and clearly, $\widetilde{\mu}\left(\cap L_{n}\right)=\inf \widetilde{\tilde{\mu}}\left(L_{n}\right)$.

The following results are generally well-known, and we list them for completeness:

If $\mu \in I(\mathcal{L})$, then

If $\mu \in J(\mathcal{L})$, then

$$
\mathcal{S}_{\mu^{\prime}} \cap \mathcal{L}=\left\{L \in \mathcal{L} \mid \mu(L)=\mu^{\prime}(L)\right\}
$$


If $\mu \in I_{\sigma}(\mathcal{L})$, and if

$$
\mathcal{S}_{\mu^{\prime \prime}} \cap \mathcal{L}=\left\{L \in \mathcal{L} \mid \mu(L)=\mu^{\prime \prime}(L)\right\}
$$

then $\mu \in J(\mathcal{L})$

$$
\mathcal{S}_{\mu^{\prime \prime}} \cap \mathcal{L}=\left\{L \in \mathcal{L} \mid \mu(L)=\mu^{\prime \prime}(L)\right\},
$$

We extend some of these results to the following

THEOREM 3.6. If $\mu \in I_{\sigma}(\mathcal{L})$, then $\mathcal{L} \subset \mathcal{S}_{\mu^{\prime \prime}}$ if and only if for every $L \in \mathcal{L}, \mu^{\prime \prime}\left(L^{\prime}\right)=1$ implies $L^{\prime} \supset \bigcap_{1}^{\infty} L_{n}, L_{n} \in \mathcal{L}, \mu\left(L_{n}\right)=1$ for all $n$

PROOF. (a) Suppose $\mu \in I_{\sigma}(\mathcal{L})$ To show that $\mathcal{L} \subset \mathcal{S}_{\mu^{\prime \prime}}$ under the above hypothesis, we need just consider two cases If $\mu^{\prime \prime}\left(L^{\prime}\right)=0$, where $L \in \mathcal{L}$, then, trivially, $L \in \mathcal{S}_{\mu^{\prime \prime}} \quad$ If $\mu^{\prime \prime}\left(L^{\prime}\right)=1$, then $L^{\prime} \supset \bigcap_{1}^{\infty}$ $L_{n}, L_{n} \in \mathcal{L}, \mu\left(L_{n}\right)=1$, and $L \in \mathcal{S}_{\mu^{\prime \prime}}$ by the results of Section 2 .

(b) Conversely, if $\mathcal{L} \subset \mathcal{S}_{\mu^{\prime \prime}}$, then again by Section 2, either

$$
L \supset \cap L_{n}, L_{n} \downarrow, L_{n} \in \mathcal{L},
$$

and $\mu\left(\mathbf{L}_{n}\right)=1$, all $n$, or $L^{\prime} \supset \cap L_{n}, L_{n} \downarrow, L_{n} \in \mathcal{L}$, and $\mu\left(L_{n}\right)=1$, all $n$. Now, if $\mu^{\prime \prime}\left(L^{\prime}\right)=1$, then, in the first case, $L^{\prime} \subset \cup L_{n}^{\prime}, \mu\left(L_{n}^{\prime}\right)=0$, all $n$, so, $\mu^{\prime \prime}\left(L^{\prime}\right)=0$. Hence, the second case must hold which completes the proof.

As an immediate consequence, we get

COROLLARY 3.6. If $\mu \in J(\mathcal{L})$ and if $\mathcal{L} \subset \mathcal{S}_{\mu^{\prime \prime}}$, then $\mu \in I_{S}(\mathcal{L})$.

Next, we recall (see [4])

DEFINITION 3.2. $\mu \in I_{w}(\mathcal{L})$ (weakly regular) if $\mu \in I(\mathcal{L})$, and if $\mu\left(L^{\prime}\right)=1, L \in \mathcal{L}$, implies $L^{\prime} \supset \tilde{L} \in \mathcal{L}$ such that $\mu^{\prime}(\tilde{L})=1$

Clearly, $I_{R}(\mathcal{L}) \subset I_{w}(\mathcal{L})$. It is not difficult to show that if $\mathcal{L}$ is normal, then $I_{w}(\mathcal{L})=I_{R}(\mathcal{L})$

We now establish the following:

THEOREM 3.7. If $\delta\left(\mathcal{L}^{\prime}\right)$ separates $\mathcal{L}$, then $\mu \in I_{\sigma}\left(L^{\prime}\right) \cap I_{w}(\mathcal{L})$ implies that $\mu \in I_{R}(\mathcal{L})$.

PROOF. Suppose $\mu\left(L^{\prime}\right)=1$, where $L \in \mathcal{L}$. Then $L^{\prime} \supset \widetilde{L} \in \mathcal{L}$ with $\mu^{\prime}(\widetilde{L})=1$, since $\mu \in I_{w}(\mathcal{L})$. Therefore, since $\delta\left(\mathcal{L}^{\prime}\right)$ separates $\mathcal{L}$, there exists $A_{2}, B_{\jmath} \in \mathcal{L}$ such that $L \subset \bigcap_{1}^{\infty} A_{2}^{\prime}, \tilde{L} \subset \bigcap_{1}^{\infty} B_{\jmath}^{\prime}$, and $\bigcap_{i, j}\left(A_{\imath}^{\prime} \cap B_{\jmath}^{\prime}\right)=\emptyset$ (may assume $\downarrow$ ). Thus, since $\mu \in I_{\sigma}\left(\mathcal{L}^{\prime}\right), \mu\left(A_{n}^{\prime} \cap B_{m}^{\prime}\right)=0, n, m>N$. But $\mu^{\prime}(\tilde{L})=1$ implies that $\mu\left(B_{m}^{\prime}\right)=1$, all $m$ So, $\mu\left(A_{n}^{\prime}\right)=0, n \geq N$. Consequently, $\mu\left(A_{n}\right)=1, n \geq N$, and $A_{n} \subset L^{\prime}$. Therefore, $\mu \in I_{R}(\mathcal{L})$. This completes the proof

DEFINITION 3.3. $\mathcal{L}$ is slightly normal if $\mu \in I_{\sigma}\left(\mathcal{L}^{\prime}\right), \mu \leq \nu_{1}(\mathcal{L}), \mu \leq \nu_{2}(\mathcal{L})$, where $\nu_{1}$, $\nu_{2} \in I_{R}(\mathcal{L})$ implies $\nu_{1}=\nu_{2}$.

THEOREM 3.8. If $\delta\left(\mathcal{L}^{\prime}\right)$ separates $\mathcal{L}$, then $\mathcal{L}$ is slightly normal.

PROOF. Suppose $\mu \in I_{\sigma}\left(\mathcal{L}^{\prime}\right), \mu \leq \nu_{1}(\mathcal{L}), \mu \leq \nu_{2}(\mathcal{L})$, where $\nu_{1}, \nu_{2} \in I_{R}(\mathcal{L})$. Suppose $\nu_{1} \neq \nu_{2}$. Then there exist $L, \tilde{L} \in \mathcal{L}$ such that $\nu_{1}(L)=\nu_{2}(\tilde{L})=1$, and $\nu_{1}(\tilde{L})=\nu_{2}(L)=0$, and $L \cap \widetilde{L}=\emptyset$. Also, by hypothesis, there exist $L_{\imath}, \widetilde{L}_{k}$ where $L_{\imath}, \widetilde{L}_{k} \in \mathcal{L}$, all $i, k$, such that $L \subset \bigcap_{1}^{\infty} L_{\imath}^{\prime}, \widetilde{L} \subset \bigcap_{1}^{\infty} \tilde{L}_{k}^{\prime}$, (and may assume $L_{\imath}^{\prime} \downarrow$ and $\left.\widetilde{L}_{k}^{\prime} \downarrow\right)$, and $\emptyset=\left(\cap L_{\imath}^{\prime}\right) \cap\left(\cap \widetilde{L}_{k}^{\prime}\right)$. Now, since $\nu_{1}(L)=1$, and $\mu \leq \nu_{1}(\mathcal{L})$, then $\mu\left(L_{\imath}^{\prime}\right)=1$, all $i$, and similarly, $\mu\left(\widetilde{L}_{\mathrm{k}}^{\prime}\right)=1$, all $k$. Therefore, $\mu\left(L_{\imath}^{\prime} \cap \widetilde{L}_{k}^{\prime}\right)=1$, all $i, k$, but $\bigcap_{i, k}$ $L_{\imath}^{\prime} \cap \tilde{L}_{k}^{\prime}=\emptyset$. Hence, we have a contradiction, since $\mu \in I_{\sigma}\left(\mathcal{L}^{\prime}\right)$. Therefore, $\nu_{1}=\nu_{2}$, and consequently, $\mathcal{L}$ is slightly normal. This completes the proof.

DEFINITION 3.4. $\mathcal{L}$ is almost normal, if $A, B \in \mathcal{L}$ and $A \cap B=\emptyset$ implies there exist $A_{\imath}^{\prime} \uparrow$, $A_{\imath} \in \mathcal{L}$ such that $A \subset \bigcup_{1}^{\infty} A_{\imath}^{\prime}$ and there exists $B_{\imath} \in \mathcal{L}$ with $A_{\imath}^{\prime} \subset B_{\imath}$, all $i$ and $B_{\imath} \cap B=\emptyset$, for all $i$.

NOTE. It is not difficult to show that if $\mathcal{L}$ is a delta lattice, and if $\mathcal{L}$ is almost normal, then $\mathcal{L}$ is normal.

We now show the following:

THEOREM 3.9. Let $\mathcal{L}$ be almost normal. Suppose $\mu \in I(\mathcal{L})$ and $\mu \leq \nu(\mathcal{L})$, where $\nu \in I_{R}^{\sigma}(\mathcal{L})$, then $\nu^{\prime \prime}=\mu^{\prime \prime}(\mathcal{L})$. 
PROOF. Since $\mu \leq \nu(\mathcal{L}), \nu^{\prime \prime} \leq \mu^{\prime \prime}$ everywhere, and consequently, $\nu^{\prime \prime} \leq \mu^{\prime \prime}(\mathcal{L}) \quad$ Let $L \in \mathcal{L}$ be such that $\nu(L)=\nu^{\prime \prime}(L)=0 \quad$ Then $\nu\left(L^{\prime}\right)=1$, and therefore, $L^{\prime} \supset \widetilde{L} \in \mathcal{L}$ with $\nu(\tilde{L})=1$, since $\nu \in I_{R}^{o}(\mathcal{L})$ Therefore, (by Definition 34 ), there exists $A_{\imath}$ such that $\tilde{L} \subset \cup A_{\imath}^{\prime}, A_{\imath}^{\prime} \uparrow, A_{\imath} \in \mathcal{L}$, and there exists $B_{\imath}$ such that $B_{\imath} \in \mathcal{L}, A_{\imath}^{\prime} \subset B_{\imath}$, all $i$, and $B_{\imath} \cap L=\emptyset$ So, for some $i_{0}, \nu\left(A_{\imath}^{\prime}\right)=1, i \geq i_{0}$ Therefore, $\mu\left(A_{\imath}^{\prime}\right)=1$, for all $i \geq i_{0} \quad$ Consequently, $\mu\left(B_{\imath}^{\prime}\right)=0$, all $i \geq i_{0} \quad$ Hence, $\mu\left(B_{\imath}^{\prime}\right)=0, i \geq i_{0}$. But, $L \subset B_{\imath}^{\prime}$, for all $i \quad$ Therefore, $\mu^{\prime}(L)=0 \quad$ Therefore, $\mu^{\prime \prime}(L)=0 \quad$ Hence, $\nu^{\prime \prime}=\mu^{\prime \prime}(\mathcal{L})$. This completes the proof.

We can extend this result even further. First, recall (see [5])

DEFINITION 3.5. $\mu \in I_{v}(\mathcal{L})$ ( $\mu$ is vaguely regular) if $\mu \in I_{\sigma}(\mathcal{L})$, and if

$$
\mu\left(L^{\prime}\right)=1, L \in \mathcal{L},
$$

then $L^{\prime} \supset \widetilde{L}, \tilde{L} \in \mathcal{L}$, and $\mu^{\prime \prime}(\tilde{L})=1$. Clearly, $I_{R}^{\sigma}(\mathcal{L}) \subset I_{v}(\mathcal{L}) \subset I_{w}(\mathcal{L})$, and it is easy to see that $I_{v}(\mathcal{L}) \subset J(\mathcal{L})$ With appropriate modifications, it is now easy to extend Theorem 3.9.

THEOREM 3.10. Let $\mathcal{L}$ be almost normal. Suppose $\mu \in I(\mathcal{L})$ and $\mu \leq \nu(\mathcal{L})$, where $\nu \in I_{v}(\mathcal{L})$. Then $\nu^{\prime \prime}=\mu^{\prime \prime}(\mathcal{L})$

THEOREM 3.11. If $\nu \in J(\mathcal{L})$, and if $\mu \leq \nu(\mathcal{L})$, and if $\mathcal{L}$ is normal and semi-separates $\delta(\mathcal{L})$, then $\nu^{\prime \prime}=\mu^{\prime \prime}(\mathcal{L})$.

PROOF. Since $\nu \in J(\mathcal{L})$, it is not difficult to see that we can extend $\nu$ uniquely to $J(\delta(\mathcal{L})$ ), and we denote the extension by $\nu$ again. Now, $\mu \leq \nu \leq \nu^{\prime \prime} \leq \mu^{\prime \prime}(\mathcal{L})$. Since $\mathcal{L}$ is normal, we have (see Section 2) $\nu^{\prime}=\mu^{\prime}(\mathcal{L})$. Suppose $L \in \mathcal{L}$ and $\nu^{\prime \prime}(L)=0$. This implies that $L \subset \bigcup_{1}^{\infty} L_{n}^{\prime}, L_{n} \in \mathcal{L}$, all $n$, and $\nu\left(L_{n}^{\prime}\right)=0$, all $n$. Therefore, $L^{\prime} \supset \bigcap_{1}^{\infty} L_{n}, \nu\left(L_{n}\right)=1$, all $n$, and consequently, $\nu\left(\bigcap_{1}^{\infty} L_{n}\right)=1$.

Now, $\bigcap L_{n} \in \delta(\mathcal{L})$ and since $\mathcal{L}$ semi-separates $\delta(\mathcal{L})$, there exists $A \in \mathcal{L}$ such that $L \cap A=\emptyset$ and $A \supset \bigcap_{1}^{\infty} L_{n}$. So, $\nu(A)=1, \nu\left(A^{\prime}\right)=0$ and $L \subset A^{\prime}$ implies $\nu^{\prime}(L)=0$. Whence, $\mu^{\prime}(L)=0$. But $\mu^{\prime \prime} \leq \mu^{\prime}$ everywhere, therefore, $\mu^{\prime \prime}(L)=0$. Hence, $\nu^{\prime \prime}=\mu^{\prime \prime}(\mathcal{L})$. This completes the proof.

As a final observation in this section, we note that the measures of $I_{S}(\mathcal{L})$ are maximal in the following sense. Let $\mu \leq \nu(\mathcal{L})$, where $\mu \in I_{S}(\mathcal{L})$ and $\nu \in I_{\sigma}(\mathcal{L})$. Then $\nu \in I_{S}(\mathcal{L})$. This is clear since $\mu \leq \nu \leq \nu^{\prime \prime} \leq \mu^{\prime \prime}(\mathcal{L})$ (see (2.1)), but $\mu=\mu^{\prime \prime}(\mathcal{L})$ since $\mu \in I_{S}(\mathcal{L})$ (by Theorem 3.1). Hence, $\nu=\nu^{\prime \prime}(\mathcal{L})$, and therefore, $\nu \in I_{S}(\mathcal{L})$. Moreover, if $\mu_{1} \leq \mu_{2}(\mathcal{L})$, and $\mu_{1}, \mu_{2} \in I_{S}(\mathcal{L})$, then $\mu_{1}=\mu_{2}$ as is easily seen. This has the following consequence. Let $\mu \in I_{S}(\mathcal{L})$, then there exists a $\nu \in I_{R}(\mathcal{L})$ such that $\mu \leq \nu(\mathcal{L})$. Now if $\mathcal{L}$ is normal and c.p., then $\nu \in I_{R}^{\sigma}(\mathcal{L})$, and, consequently, $\mu=\nu$. Hence if $\mathcal{L}$ is normal and c.p., then $I_{S}(\mathcal{L})=I_{R}^{\sigma}(\mathcal{L})$.

\section{FURTHER APPLICATIONS ON REGULARITY.}

In this section, we consider applications of the associated outer measures to regularity properties.

If $\mu \in I^{\sigma}(\mathcal{L})$, and, for $E \subset X$, we define the usual induced outer measure:

$$
\mu^{*}(E)=\inf \left\{\sum_{\imath=1}^{\infty} \mu\left(A_{\imath}\right) \mid E \subset \bigcup_{1}^{\infty} A_{\imath}, A_{\imath} \in \mathcal{A}(\mathcal{L})\right\}
$$

We note trivially that if $\mu \in I_{R}^{\sigma}(\mathcal{L})$, then $\mu^{*}=\mu^{\prime \prime}$, and if, in addition, $\mathcal{L}$ is a delta lattice then $\mu^{*}=\mu^{\prime \prime}=\mu^{\prime}$.

We consider those $\mu \in I_{\sigma}(\mathcal{L})$ which satisfy the following condition:

(i) $\mu^{\prime \prime}\left(L^{\prime}\right)=1$ implies there exists an $\widetilde{L} \subset L^{\prime}$ with $\mu(\widetilde{L})=1$, where $L, \widetilde{L} \in \mathcal{L}$.

THEOREM 4.1. Let $\mu \in I_{\sigma}(\mathcal{L})$, and let $\mu$ satisfy condition (i) Then $\mathcal{L} \subset \mathcal{S}_{\mu^{n}}$ and $\left.\mu^{\prime \prime}\right|_{\mathcal{A}(\mathcal{L})} \in I_{R}^{\sigma}(\mathcal{L})$.

PROOF. Suppose $\mu \in I_{\sigma}(\mathcal{L})$, and $\mu$ satisfies condition (i). Then if $L \in \mathcal{L}$, and if $\mu^{\prime \prime}\left(L^{\prime}\right)=0$ then $L^{\prime} \in \mathcal{S}_{\mu^{\prime \prime}}$. Therefore, $L \in \mathcal{S}_{\mu^{\prime \prime}}$. Also, if $\mu^{\prime \prime}\left(L^{\prime}\right)=1$ then $L^{\prime} \supset \tilde{L} \in \mathcal{L}$, and $\mu(\tilde{L})=1$, by condition (i), but $L^{\prime} \supset \tilde{L} \in \mathcal{L}$ and $\mu(\widetilde{L})=1$ implies $L \in \mathcal{S}_{\mu^{\prime}} \subset \mathcal{S}_{\mu^{\prime \prime}}$. Therefore, $\mathcal{L} \subset \mathcal{S}_{\mu^{\prime \prime}}$, and consequently, 
$\left.\mu^{\prime \prime}\right|_{\mathcal{A}(\mathcal{L})} \in I^{\sigma}(\mathcal{L})$ and $\mu \leq \mu^{\prime \prime}(\mathcal{L}) \quad$ Also, $\mu^{\prime \prime}\left(L^{\prime}\right)=1$ implies $L^{\prime} \supset \widetilde{L}, \mu(\tilde{L})=1, \tilde{L} \in \mathcal{L} \quad$ Hence, $\mu^{\prime \prime}(\tilde{L})=1$ and $\left.\mu^{\prime \prime}\right|_{\mathcal{A}(\mathcal{L})} \in I_{R}^{\sigma}(\mathcal{L})$

For $\mu \in I_{\sigma}(\mathcal{L})$, we also consider the following weaker condition.

(ii) $\mu^{\prime \prime}\left(L^{\prime}\right)=1$ implies there exists $\widetilde{L} \subset L^{\prime}$ with $\mu^{\prime \prime}(\tilde{L})=1$, where $L, \widetilde{L} \in \mathcal{L}$

We then have

THEOREM 4.2. If $\mu \in I_{\sigma}(\mathcal{L})$ and if $\mathcal{L} \subset \mathcal{S}_{\mu^{\prime \prime}}$, then $\left.\mu^{\prime \prime}\right|_{\mathcal{A}(\mathcal{L})} \in I^{\sigma}(\mathcal{L})$, and if $\mu$ satisfies condition (ii), then $\left.\mu^{\prime \prime}\right|_{\mathcal{A}(\mathcal{L})} \in I_{R}^{o}(\mathcal{L})$

PROOF. The proof is clear

In the same spirit, we have

THEOREM 4.3. If $\mu \in I_{\sigma}(\mathcal{L})$, and if $\mathcal{L} \subset \mathcal{S}_{\mu^{\prime \prime}}$, and if $\mathcal{L}$ semi-separates $\delta(\mathcal{L})$, then $\left.\mu^{\prime \prime}\right|_{\mathcal{A}(\mathcal{L})} \in I_{R}^{\sigma}(\mathcal{L})$

PROOF. Suppose $\mu \in I_{\sigma}(\mathcal{L}), \mathcal{L} \subset \mathcal{S}_{\mu^{\prime \prime}}, \mathcal{L}$ semi-separates $\delta(\mathcal{L})$ and $\mu^{\prime \prime}\left(L^{\prime}\right)=1, L \in \mathcal{L}$ Then $\mu^{\prime \prime}(L)=0$, since $\mathcal{L} \subset \mathcal{S}_{\mu^{\prime \prime}} \quad$ Therefore, there exists $L_{n} \in \mathcal{L}$ such that $L \subset \cup L_{n}^{\prime}$ and $\mu\left(L_{n}^{\prime}\right)=0$, all $n$. Hence, $L^{\prime} \supset \cap L_{n}, \mu\left(L_{n}\right)=1$, all $n$. By semi-separation, $\bigcap_{1}^{\infty} L_{n} \subset \widetilde{L} \in \mathcal{L}$, and $L \cap \widetilde{L}=\emptyset$ Therefore, $\mu^{\prime \prime}\left(L_{n}\right)=1$ and $\mu^{\prime \prime}\left(\cap L_{n}\right)=1$, since $\mathcal{L} \subset \mathcal{S}_{\mu^{\prime \prime}}$. Thus, $\mu^{\prime \prime}(\tilde{L})=1$. Now $\mu \leq\left.\mu^{\prime \prime}\right|_{\mathcal{A}(\mathcal{L})} \in I^{\sigma}(\mathcal{L})$ clearly.

COROLLARY 4.3. If $\mu \in J(\mathcal{L})$, and if $\mathcal{L} \subset \mathcal{S}_{\mu^{n}}$, and if $\mathcal{L}$ semi-separates $\delta(\mathcal{L})$, then $\mu \in I_{R}^{o}(\mathcal{L})$

PROOF. We note that this follows easily from Corollary 36 , Theorem 31 (a), and Theorem 43.

Next, we note some measurability conditions.

THEOREM 4.4. (1) Let $\mu \in I_{\sigma}\left(\mathcal{L}^{\prime}\right)$ and let $A \in \mathcal{L}$ and $A=\bigcap_{1}^{\infty} B_{n}^{\prime}, B_{n} \in \mathcal{L}$. Then $A \in \mathcal{S}_{\widetilde{\mu}}$.

(2) Let $\mu \in I_{\sigma}(\mathcal{L})$, and let $\mathcal{L}$ be normal Then if $A \in \mathcal{L}$, and if $A=\bigcap_{1}^{\infty} B_{n}^{\prime}, B_{n} \in \mathcal{L}$, all $n$. Then $A \in \mathcal{S}_{\mu^{\prime \prime}}$.

PROOF. (1) If $\widetilde{\mu}(A)=0$, then, clearly, $A \in \mathcal{S}_{\tilde{\mu}}$. Suppose $\widetilde{\mu}(A)=1$. Then $\mu(A)=1$ since $\widetilde{\tilde{\mu}} \leq \mu(\mathcal{L})$ (by 2.2, Section 2). Now, $A=\bigcap_{1}^{\infty} B_{n}^{\prime}$, and $A \subset B_{n}^{\prime}$, all $n$, and $\mu(A)=1$ implies $\mu\left(B_{n}^{\prime}\right)=1$, all $n$. Therefore, $\mathcal{A} \in \mathcal{S}_{\widetilde{\tilde{\mu}}}$.

(2) Suppose $\mu \in I_{\sigma}(\mathcal{L})$, and $\mathcal{L}$ normal. Suppose $A=\cap B_{n}^{\prime}, A \in \mathcal{L}, B_{n} \in \mathcal{L}$, all $n$. Now, if $\mu^{\prime \prime}(A)=0$, then we're done; while if $\mu^{\prime \prime}(A)=1$, then $\mu^{\prime \prime}\left(B_{n}^{\prime}\right)=1$, all $n$. By normality, $A \subset C_{n}^{\prime} \subset D_{n} \subset B_{n}^{\prime}, C_{n}, D_{n} \in \mathcal{L}$, all $n$. Therefore, $A=\cap D_{n}$, and $\mu^{\prime \prime}(A)=1$ implies $\mu^{\prime \prime}\left(C_{n}^{\prime}\right)=1$. Hence, $\mu\left(C_{n}^{\prime}\right)=1$ (since $\mu^{\prime \prime} \leq \mu\left(\mathcal{L}^{\prime}\right)$ ), and consequently, $\mu\left(D_{n}\right)=1$, all $n$. Thus $A \in \mathcal{S}_{\mu^{\prime \prime}}$ (see Section 2). This completes the proof.

REMARK. We note, by part 1 of Theorem 4.4, that if $\mu \in I_{\sigma}\left(\mathcal{L}^{\prime}\right)$ and if $\mathcal{L}$ is c.g., then $\mathcal{L} \subset \mathcal{S}_{\tilde{\mu}}$. Also, if $\mu \in I_{\sigma}(\mathcal{L})$ and if $\mathcal{L}$ is c.g., then it is easy to see that condition (ii) is satisfied, which gives an alternate approach to Theorem 2.2 .

We conclude this section with some remarks on the Wallman space $I(\mathcal{L}), V(\mathcal{L})$. We recall (see [2]), for $A \in \mathcal{A}(\mathcal{L}), V(A)=\{\mu \in I(\mathcal{L}) \mid \mu(A)=1\}$. Then for

$$
\begin{aligned}
A, B \in \mathcal{A}(\mathcal{L}): V(A \cup B) & =V(A) \cup V(B), V(A \cap B)=V(A) \cap V(B), \\
V\left(A^{\prime}\right) & =V(A)^{\prime} \quad \text { and } \quad V(A) \subset V(B)
\end{aligned}
$$

if and only if $A \subset B, V(\mathcal{A}(\mathcal{L}))=\mathcal{A}(V(\mathcal{L}))$, where $V(\mathcal{L})=\{V(L) \mid L \in \mathcal{L}\}$.

For $\mu \in I(\mathcal{L})$, we define a set function $\tilde{\mu}$ on $\mathcal{A}(V(\mathcal{L}))$ by $\bar{\mu}(V(A))=\mu(A)$ for $A \in \mathcal{A}(\mathcal{L})$. This sets up a bijection between $I(\mathcal{L})$ and $I(V(\mathcal{L}))$.

From the above, it readily follows that $V(\mathcal{L})$ is a base for the closed sets of a topology. $\tau V(\mathcal{L})$ designates the closed sets of the topology and consists of all arbitrary intersections of sets of $V(\mathcal{L})$. Then $I(\mathcal{L})$ with this topology is compact, $T_{0}$.

Now, we denote, for $x \in X$, by $\mu_{x}$, the Dirac measure concentrated at $x$, i e.,

$$
\mu_{x}(A)= \begin{cases}1 & \text { if } x \in A \\ 0 & \text { if } x \notin A \text { where } \quad A \in \mathcal{A}(\mathcal{L}) .\end{cases}
$$


If $\mathcal{L}$ is $T_{0}$ then the map $x \rightarrow \mu_{x}$, embeds $X$ in $I(\mathcal{L})$ We assume now that $\mathcal{L}$ is $T_{0}$, and we have:

THEOREM 4.5. (1) If $\mu \in I_{\sigma}(\mathcal{L})$, and if $\cap V\left(L_{n}\right) \subset I(\mathcal{L})-X$, where $V\left(L_{n}\right) \downarrow, L_{n} \in \mathcal{L}$, then $\bar{\mu}\left(V\left(L_{n}\right)\right) \rightarrow 0$

(2) $\mu \in J(\mathcal{L})$ implies $\bar{\mu} \in J(V(\mathcal{L}))$

(3) $\mu \in I(\mathcal{L})$, then $\bar{\mu}^{\prime}(V(L))=\mu^{\prime}(L)$

PROOF. (1) Let $\mu \in I_{\sigma}(\mathcal{L})$, and let $\bigcap_{1}^{\infty} V\left(L_{n}\right) \subset I(\mathcal{L})-X$, where $L_{n} \in \mathcal{L}$, and $V\left(L_{n}\right) \downarrow$. Then clearly $L_{n} \downarrow \emptyset$ and $\bar{\mu}\left(V\left(L_{n}\right)\right)=\mu\left(L_{n}\right) \rightarrow 0$.

(2) Let $\mu \in J(\mathcal{L})$ and let $V\left(L_{n}\right) \downarrow V(L)$ where $L_{n}, L \in \mathcal{L}$ then $L_{n} \downarrow L$, and consequently, $\bar{\mu}\left(V\left(L_{n}\right)\right)=\mu\left(L_{n}\right) \rightarrow \mu(L)=\bar{\mu}(V(L))$.

(3) The proof is clear.

Since $V(\mathcal{L})$ is a compact lattice, it is certainly c c and, hence,

$$
I(V(\mathcal{L}))=I_{\sigma}(V(\mathcal{L}))
$$

Thus, to obtain a characterization of those $\mu \in I_{\sigma}(\mathcal{L})$ in terms of the associated $\bar{\mu}$, we must look elsewhere.

In fact, we have:

THEOREM 4.6. (1) Let $\mu \in I(\mathcal{L})$. If $\bar{\mu}^{\prime}\left(\cap V\left(L_{n}\right)\right)=0$, for all $\cap V\left(L_{n}\right) \subset I(\mathcal{L})-X$, where $V\left(L_{n}\right) \downarrow, L_{n} \in \mathcal{L}$, then $\mu \in I_{\sigma}(\mathcal{L})$, and $L_{n} \in \mathcal{S}_{\mu^{\prime}}$ for all $n$ sufficiently large.

(2) Conversely, if $\mu \in I_{\sigma}(\mathcal{L})$, and if $\cap V\left(L_{n}\right) \subset I(\mathcal{L})-X$, where $V\left(L_{n}\right) \downarrow, L_{n} \in \mathcal{L}$, and if $L_{n} \in \mathcal{S}_{\mu^{\prime}}$ for all $n \geq N$, then $\bar{\mu}^{\prime}\left(\cap V\left(L_{n}\right)\right)=0$.

PROOF. (1) Let $L_{n} \in \mathcal{L}$, and $L_{n} \downarrow \emptyset$. Then $\cap V\left(L_{n}\right) \subset I(\mathcal{L})-X$ and $V\left(L_{n}\right) \downarrow$. Thus, $\bar{\mu}^{\prime}\left(\cap V\left(L_{n}\right)\right)=0$. Hence, there exists

$$
L \in \mathcal{L}, \quad \cap V\left(L_{n}\right) \subset V(L)^{\prime}, \bar{\mu}\left(V(L)^{\prime}\right)=0, \bar{\mu}(V(L))=1, \mu(L)=1, \mu\left(L^{\prime}\right)=0 .
$$

Now $\cap V\left(L_{n}\right) \cap V(L)=\emptyset$. Hence, $\cap V\left(L_{n} \cap L\right)=\emptyset$ implies that $V\left(L_{n} \cap L\right)=\emptyset, n \geq N$, since $V(\mathcal{L})$ is compact. But, $V\left(L_{n} \cap L\right)=\emptyset$ implies $L_{n} \cap L=\emptyset$. Whence, $L_{n} \subset L^{\prime}, n \geq N$ and $\mu\left(L_{n}\right)=0$, $n \geq N$. Therefore, $\mu \in I_{\sigma}(\mathcal{L})$, and $L_{n} \subset L^{\prime}, n \geq N, \mu^{\prime}\left(L_{n}\right) \leq \mu^{\prime}\left(L^{\prime}\right)=\mu\left(L^{\prime}\right)=0$. So, $\mu^{\prime}\left(L_{n}\right)=0$, $n \geq N$, and $L_{n} \in \mathcal{S}_{\mu^{\prime}}, n \geq N$. Hence, $\mu \in I_{\sigma}(\mathcal{L})$, and $L_{n} \in \mathcal{S}_{\mu^{\prime}}, n \geq N$.

(2) Suppose $\bar{\mu}^{\prime}\left(\cap V\left(L_{n}\right)\right)=1$. Then $\bar{\mu}^{\prime}\left(V\left(L_{n}\right)\right)=1$, all $n$. But, then $\mu^{\prime}\left(L_{n}\right)=1$, all $n$ (Theorem 4.5 part (3)). But $\mu^{\prime}\left(L_{n}\right)=\mu\left(L_{n}\right)$, for $n \geq N$, by (3.1). Therefore, $\mu\left(L_{n}\right)=1$, all $n \geq N$, a contradiction, since $\mu \in I_{\sigma}(\mathcal{L})$. Therefore, $\bar{\mu}^{\prime}\left(\cap V\left(L_{n}\right)\right)=0$. This completes the proof.

ACKNOWLEDGMENT. The author would like to thank the referees for their help in improving the presentation of the paper.

\section{REFERENCES}

[1] ALEXANDROFF, A.D., Additive set functions in abstract spaces, Math. Sb. (N.S) 8, 50 (1940), 307-348.

[2] BACHMAN, G. and STRATIGOS, P.D., On general lattice repleteness and completeness, Illinois J. Math. 27 (1983), No. 4, 535-561.

[3] FROLIK, Z., Prime filters with the c.i.p., Comm. Math. Univ. Carolina, 13, 3 (1972), 553-575.

[4] GRASSI, P., Outer measures and associated lattice properties, Internat. J. Math. \& Math. Sci. 16 No. 4 (1993), 687-694.

[5] SIEGEL, D., Outer measures and weak regularity of measures (to appear Internat. J. Math. \& Math. Sci.).

[6] SZETO, M., On separation of lattices, Internat. J. Math. \& Math. Sci. 14, No. 2 (1991), 325-338.

[7] SZETO, M., On normal lattices and separation properties of lattices, Journal of the Indian Math. Soc., 58, No. 1 (1992), 51-64.

[8] WALLMAN, H., Lattices and topological spaces, Ann. of Math. 39 (1938), 112-126. 


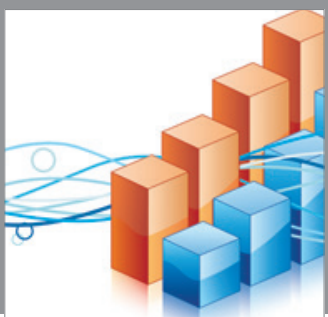

Advances in

Operations Research

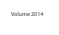

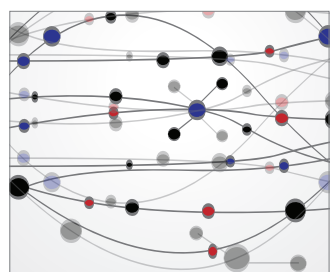

\section{The Scientific} World Journal
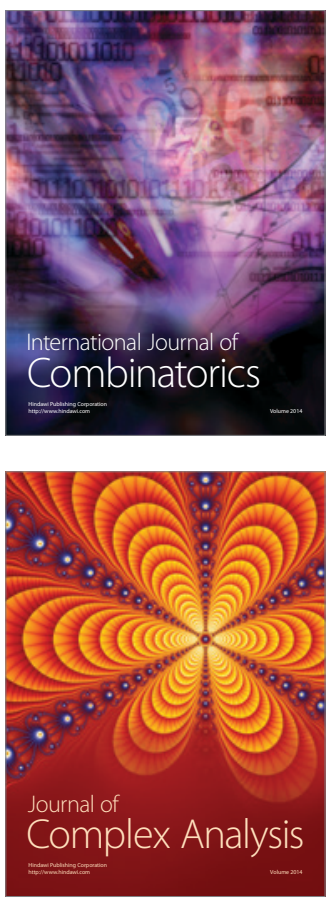

International Journal of

Mathematics and

Mathematical

Sciences
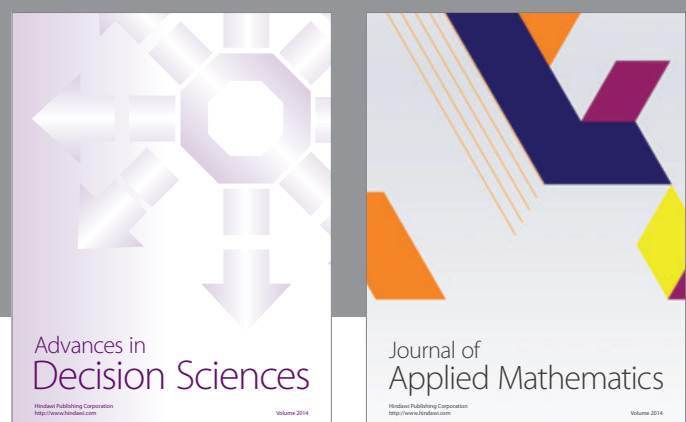

Journal of

Applied Mathematics
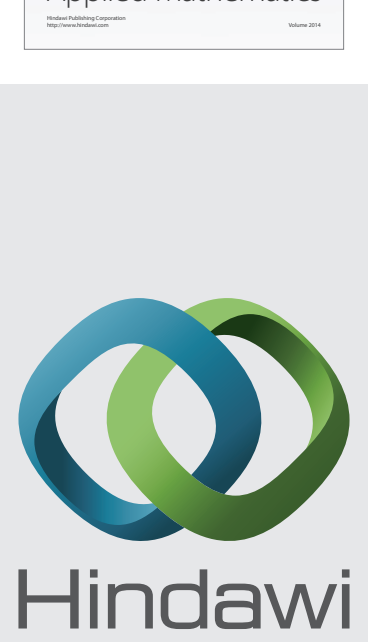

Submit your manuscripts at http://www.hindawi.com
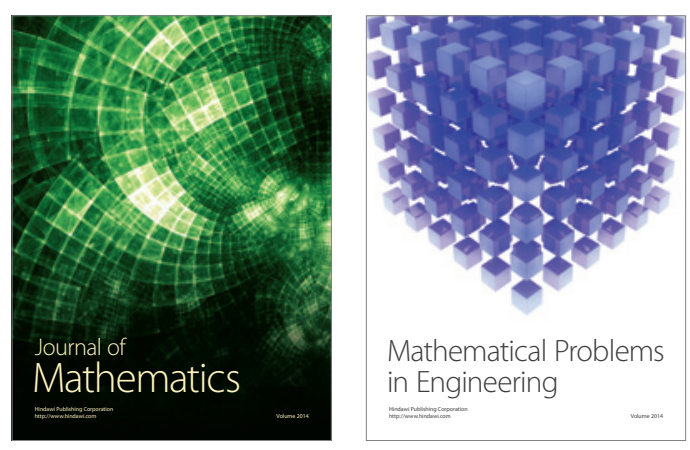

Mathematical Problems in Engineering
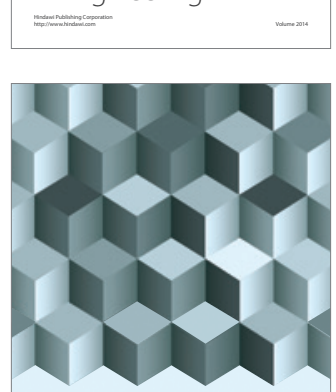

Journal of

Function Spaces
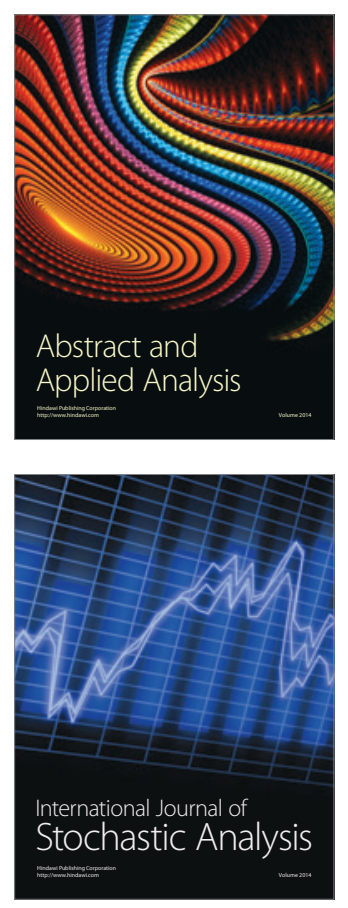

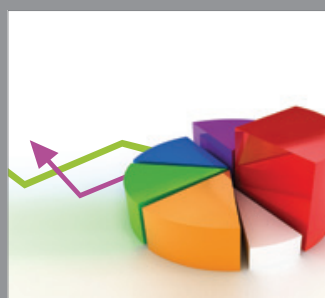

ournal of

Probability and Statistics

Promensencen
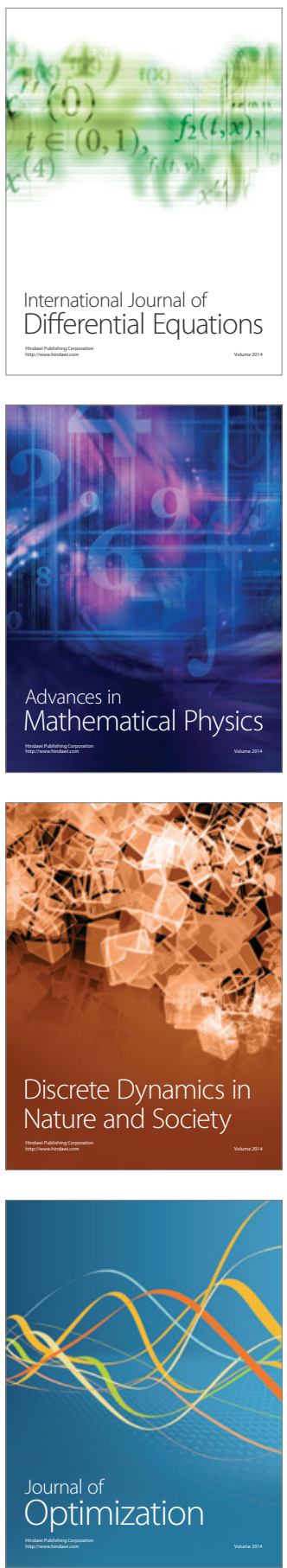